\title{
Research on Performance Measuring and Follow-up Response Strategy for Transformation of Resource-based Cities: A Case Study of Huangshi in Central China's Hubei Province
}

\author{
Zutao Hao ${ }^{1, a}$, Bing Feng ${ }^{1, b}$, Zhanyong WU ${ }^{2, c}$, Ying Wang ${ }^{3, d}$ \\ ${ }^{1}$ School of Management, Wuhan Institute of Technology, Wuhan 430205, China \\ 2 School of Management, Nanyang Institute of Technology, Nanyang, 473004, China \\ ${ }^{3}$ School of Economics and Management, China University of Geosciences, Wuhan, 430074, China \\ ahaozutao@163.com, bfengtao7168@163.com, cwuzhany@163.com, ‘wangying@cug.edu.cn
}

\begin{abstract}
Keywords: resource-based cities; transformation; performance measuring; response strategy Abstract. This paper constructs an index system for the measurement of the transformation performance of resource-based cities, and weight of each indicator is determined as per the expert group decision-making method on the basis of Analytic Hierarchy Process model (AHP). Further,the paper combines tangible indicator of material output with intangible indicator of people satisfaction. Then, taking Huangshi City in Central China's Hubei Province as an example, the transformation performance of resource-based cities is measured. According to the measurement, the score of transformation performance of Huangshi City is 4.13, a result in the ecellent rank, suggesting that the transformation made remarkable achievement as expected. Meanwhile, some local indicators underperformed and needs us to make relevant institutional arrangements as per the ranking of the scores of all the indicators in our future transformation work, especially the indicators with low performance scores.
\end{abstract}

\section{Introduction}

The transformation of resource-based cities is such a strategically important target that Chinese governments give abundant policy and financial support with increasing input in every year.To clearly understand the effectiveness of the transformation work of resource-based cities, we need to make scientific and reasonable measurement of the transformation performance already achieved so as to provide valid supporting reference to the further transformation of such cities and to the improvement of national supporting policy.

Measurement of the transformation performance of resource-based cities is beneficial to the implementation of the strategy of transformational development and innovation of regulatory supervision, to the increase of the utilization efficiency of fiscal transfer payment, and to the mobilization of the activity and initiative of such cities. Currently, omestic and foreign scholars mainly construct the index system for the measurement of transformation performance of resource-based cities from the perspectives of economy, society, resource and environment etc [1-3], and explore the effectiveness of transformation and relevant response strategy with SPSS, MATLAB and other tools on the bases of different mathematical models. The DEA model [4], the BP-neural network model [5], and the SEM model [6] are commonly used models, while the cross-evaluation method [4], the entropy method[7], the coefficient of variation method [7] and the hierarchy analysis method [7] are popular research methods. Current researches mostly focus on the performance of tangible material output while ignore that of the intangible subjective perception. Given the above circumstances, this paper, in taking Huangshi City in Central China's Hubei Province as an example, measures both the tangible material output and the intangible perception performance of people satisfaction so as to make more scientific and effective measurement of the transformation performance of the resource-based cities.

\section{Selection of method to assign weight to indicators}

In our research, we use the AHP method to assign weight to the indicators, and use the expert group 
decision-making method to effectively reduce the error of arbitrary grading of experts. The process of calculation is as follows:

1)Construction of judgement matrix. The experts group define the degree of importance of all indicators as per the $1 \sim 9$ demarcation method as shown in the undermentioned Table 1.

Tab.1 1-9 scale of the index importance

\begin{tabular}{cc}
\hline$a_{i j}$ value & definition \\
\hline$a_{i j}=1$ & $A_{i}$ is equally important with $A_{j}$ \\
$a_{i j}=3$ & $A_{i}$ is slightly more important than $A_{j}$ \\
$a_{i j}=5$ & $A_{i}$ is obviously more important than $A_{j}$ \\
$a_{i j}=7$ & $A_{i}$ is much more important than $A_{j}$ \\
$a_{i j}=9$ & $A_{i}$ is extremely more important than $A_{j}$ \\
$a_{i j}=2,4,6,8$ & lie between the above two circumstances
\end{tabular}

the reciprocal of above numbers reverse comparison of two elements

Compare the degree of importance of any one element of $A_{1}, A_{2}, \ldots, A_{n}$ in the $i$ layer to $B_{k}$ in the neighboring upper layer (the $i-1$ layer), use $a_{i j}$ to describe the relative degree of importance of $A_{i}$ and $A_{j}$ to $B_{k}$, and present all the results in the form of $B-A$ judgement matrix.

$$
B-A=\left[\begin{array}{ccccc}
1 & a_{12} & a_{13} & \cdots & a_{1 n} \\
1 / a_{12} & 1 & a_{23} & \cdots & a_{2 n} \\
\cdots & \cdots & \cdots & \cdots & \cdots \\
\cdots & \cdots & \cdots & \cdots & \cdots \\
1 / a_{1 n} & 1 / a_{2 n} & 1 / a_{3 n} & \cdots & 1
\end{array}\right]
$$

Where, the matrix element $a_{i j}$ has the following nature: (1) $a_{i i}=1$, namely, all the elements on the diagonal line in the matrix are $1 ;(2) a_{i j}=1 / a_{j i}$, namely, $a_{i j}$ and $a_{j i}$ are mutual opposite numbers.

2)Standardization of judgement matrix. Standardize the element of $a_{i j}$ in the judgement matrix $B-A$ as per the column it belongs to and get a new matrix $\bar{A}=\left(\bar{a}_{i j}\right)_{n \times n}$, where

$$
\bar{a}_{i j}=\frac{a_{i j}}{\sum_{i=1}^{n} a_{i j}} \quad(i, j=1,2, \cdots, n)
$$

3)Solvation of feature vector. Add the element of $\bar{a}_{i j}$ in each row in matrix $\bar{A}$ :

$$
\bar{b}_{i}=\sum_{j=1}^{n} \bar{a}_{i j} \quad(i=1,2, \cdots, n)
$$

Where elements $\bar{b}_{1} 、 \bar{b}_{2} 、 \ldots \bar{b}_{n}$ constitute matrix $\bar{B}=\left(\bar{b}_{i}\right)_{n \times 1}$, calculate the arithmetic mean value of all the elements in matrix $\bar{B}$ :

$$
\bar{w}_{i}=\frac{\bar{b}_{i}}{n} \quad(i=1,2, \cdots, n)
$$

Consequently, we get a column vector $\bar{W}=\left(\bar{w}_{1}, \bar{w}_{2}, \cdots, \bar{w}_{n}\right) \quad(i=1,2, \cdots, n)$, standardize the column vector: 


$$
w_{i}=\frac{\bar{w}_{i}}{\sum_{i=1}^{n} \bar{w}_{i}} \quad(i=1,2, \cdots, n)
$$

The resulting row vector $W=\left(w_{1}, w_{2}, \cdots, w_{n}\right)^{T} \quad(i=1,2, \cdots, n)$ is thus the target feature vector.

4)Calculation of the maximal characteristic root of judgment matrix. The computational formula to calculate the maximal characteristic root $\lambda_{\max }$ of judgment matrix is as follows:

$$
\lambda_{\max }=\sum_{i}^{n} \frac{(A W)_{i}}{n w_{i}}
$$

5)Verification of consistency. The computational formula of the consistency indicator $C I$ is:

$$
C I=\frac{\lambda_{\max }-n}{n-1}
$$

The following formula can be used to calculate the Consistency Ratio:

$$
C R=\frac{C I}{R I}
$$

As for the mean random consistency indicator $R I$, there exist correspondence relationship as demonstrated in Table 2.

Tab. 2 Values of various order RI

\begin{tabular}{cccccccccccc}
\hline order & 1 & 2 & 3 & 4 & 5 & 6 & 7 & 8 & 9 & 10 & 11 \\
\hline RI & 0.00 & 0.00 & 0.58 & 0.90 & 1.12 & 1.24 & 1.32 & 1.41 & 1.45 & 1.49 & 1.51 \\
\hline
\end{tabular}

6)Assignment of weight to indicators. Upon computation, the feature vector of the matrix $B-A$ can be determined and consistency can be verified. Accordingly, the weight of indicators is assigned.

\section{Measurement of transformation performance of resource-based cities}

\section{Data source}

In this paper, the material output data is sourced from the 2015 Statistical Bulletin of Huangshi City, the people satisfaction data come from the interviews and field surveys of the experts group of the Research Center for Coordinated Development between Enterprises and Environment of Wuhan Institute of Technology conducted in Daye,Huangshi City in May 2015.

The survey questionnaire adopts the Likert's five-point scale to category the measurement results into five grades:"quite satisfactory", "relatively satisfactory", "ordinary", "unsatisfactory" and "quite unsatisfactory". The value of the five grades are respectively weighted as: "5 points", "4 points", "3 points", "2 points" and "1 points". A total of 110 valid questionnaires were collected in the survey at last.

\section{Measurement of material output performance}

We construct an index system for the measurement of material output performance of resource-based cities, which includes 3 level-one indicators as economic development, livelihood improvement and environmental improvement, and 22 subordinate indicators. with the help of the expert group decision-making method and AHP model, we calculate the weight of each indicator to be assigned as follows: Economic development $\left(U_{1}, 1 / 3\right)$, Percentage of total output of leading resource mining industry $\left(U_{11}, 0.22\right)$, Added value of the tertiary industry $\left(U_{12}, 0.22\right)$, Ratio of R\&D investment to $\operatorname{GDP}\left(U_{13}, 0.22\right)$, Energy consumption per CNY ten thousand added value of the industrial production of enterprises above designated size $\left(U_{14}, 0.18\right)$, Ratio of fiscal revenue to $\operatorname{GDP}\left(U_{15}, 0.16\right)$; Livelihood improvement $\left(U_{2}, 1 / 3\right)$, Persons employed in urban areas $\left(U_{21}, 0.15\right)$, Disposable income per capita of urban residents $\left(U_{22}, 0.15\right)$, Coverage of minimum subsistence guarantee of urban residents $\left(U_{23}, 0.15\right)$, Number of insured of basic endowment insurance in urban areas $\left(U_{24}, 0.10\right)$, Number of insured of basic medical insurance in urban areas $\left(U_{25}, 0.15\right)$, Shantytown redevelopment area and task completion rate $\left(U_{26}, 0.10\right)$, Ratio of fiscal expenditure for livelihood( $\left.U_{27}, 0.20\right)$; Environmental 
improvement $\left(U_{3}, 1 / 3\right)$, Total emission of major pollutants( $\left.U_{31}, 0.14\right)$, Urban sewage treatment $\operatorname{rate}\left(U_{32}, 0.12\right)$, Ratio of number of days with excellent air quality $\left(U_{33}, 0.10\right)$, Ratio of comprehensive utilization and disposal of common industrial solid waste $\left(U_{34}, 0.08\right)$, Ratio of restoration of geological environment of developed mines $\left(U_{35}, 0.08\right)$, Rate of reclamation of damaged land $\left(U_{36}, 0.08\right)$, Yearly total investment in environmental pollution treatment $\left(U_{37}, 0.14\right)$, Rate of reclamation of mine goaf land $\left(U_{38}, 0.08\right)$, Ratio of total output of high-tech industry $\left(U_{39}, 0.10\right)$, Ratio of treatment of heavy metal pollution of land $\left(U_{3(10)}, 0.08\right)$.

Taking the 2015 Statistical Bulletin of Huangshi City as the base data, we calculate the rate of improvement of all the indicators of Huangshi City in 2015 against that of the 2014. Further, we calculate the material output performance of Huangshi City based on the overall rate of improvement with the help of the following formula:

$$
R=\sum_{i=1}^{3} \varphi_{i} R_{i}=\varphi_{1}\left(\sum_{j=1}^{5} r_{1 j} \varphi_{1 j}\right)+\varphi_{2}\left(\sum_{j=1}^{7} r_{2 j} \varphi_{2 j}\right)+\varphi_{3}\left(\sum_{j=1}^{10} r_{3 j} \varphi_{3 j}\right)
$$

Where, $R_{1}=\sum_{j=1}^{5} r_{1 j} \varphi_{1 j}, R_{2}=\sum_{j=1}^{7} r_{2 j} \varphi_{2 j}, R_{3}=\sum_{j=1}^{10} r_{3 j} \varphi_{3 j}, \varphi_{i j}$ and $r_{i j}$ respectively are the weight and rate of improvement of indicator $U_{i j}, \varphi_{i}$ and $R_{i}$ respectively are the weight and rate of improvement of indicator $U_{i}$, namely, the value of the rate of improvement of the indicator $U_{i}$ in 2015 as against 2014.

Upon computation, the rate of improvement of the overall transformation performance of Huangshi City in 2015 is $R=4.11 \%$, where in the indicator of economic development, the indicator of livelihood improvement and the rate of improvement of the indicator of environmental improvement respectively are $R_{1}=4.37 \%, R_{2}=4.18 \%$ and $R_{3}=3.79 \%$.

\section{Measurement of people satisfaction performance}

We construct an index system for the measurement of people satisfaction performance of resource-based cities, which includes 4 level-one indicators as resident livelihood, public service, living environment and cultural consumption, and 15 subordinate indicators. Based on the AHP model, we calculate the weight of each indicator to be assigned as follows: Residents livelihood $\left(V_{1}, 0.41\right)$, Satisfaction degree of economic income $\left(V_{11}, 0.35\right)$, Satisfaction degree of living condition $\left(V_{12}, 0.20\right)$, Satisfaction degree of employment $\left(V_{13}, 0.30\right)$, Satisfaction degree of consumer price $\left(V_{14}, 0.15\right)$; Public service $\left(V_{2}, 0.29\right)$, Satisfaction degree of administration efficiency $\left(V_{21}, 0.09\right)$, Satisfaction degree of education $\left(V_{22}, 0.18\right)$, Satisfaction degree medical treatment $\left(V_{23}, 0.26\right)$, Satisfaction degree of social security $\left(V_{24}, 0.32\right)$, Satisfaction degree of infrastructure $\left(V_{25}, 0.15\right)$;Satisfaction degree of living environment $\left(V_{3}, 0.20\right)$, Satisfaction degree of ecological environment $\left(V_{31}, 0.28\right)$, Satisfaction degree of food safety $\left(V_{32}, 0.38\right)$, Satisfaction degree of public security $\left(V_{33}, 0.34\right)$; Cultural consumption $\left(V_{4}, 0.10\right)$, Satisfaction degree of entertainment and leisure $\left(V_{41}, 0.43\right)$, Satisfaction degree of sports service $\left(V_{42}, 0.34\right)$, Satisfaction degree of tourist consumption $\left(V_{43}, 0.23\right)$.

We obtain the subjective evaluation data upon recovery and classification of the questionnaires for the livelihood satisfaction survey, which is further measured by statistical analysis with the help of the following formula:

$$
\begin{aligned}
& p_{i j}=\sum_{k=1}^{n} r_{i j k} / n \\
& P=\sum_{i=1}^{4} w_{i} P_{i}=w_{1}\left(\sum_{j=1}^{4} p_{1 j} \times w_{1 j}\right)+w_{2}\left(\sum_{j=1}^{5} p_{2 j} \times w_{2 j}\right)+w_{3}\left(\sum_{j=1}^{3} p_{3 j} \times w_{3 j}\right)+w_{4}\left(\sum_{j=1}^{3} p_{4 j} \times w_{4 j}\right)
\end{aligned}
$$


Where, $p_{i j}$ is the mean value of the satisfaction degree of the performance indicator $V_{i j}, r_{i j k}$ is the value of satisfaction degree of the performance indicator $V_{i j}$ given by the survey subject $k, n$ is the total number of valid samples, $w_{i j}$ is the weight of indicators $(i=1,2,3,4), P_{i}$ and $w_{i}$ respectively are the value of satisfaction degree and indicator weight of $V_{i}$, and $P$ is the value of people satisfaction of transformation performance of resource-based cities.

Upon calculation, the overall people satisfaction of transformation performance of Huangshi City is $P=4.14$, wherein the indicators of resident livelihood, public service, living environment and cultural consumption respectively are $P_{1}=4.45, P_{2}=3.82, P_{3}=3.97$ and $P_{4}=4.18$

\section{Measurement of overall transformation performance}

To get a more scientific and effective measurement of the transformation performance of resource-based cities, we add the intangible indicator of people satisfaction in addition to the tangible material output. The overall performance of transformation of Huangshi City is calculated as follows:

$$
O P=R \times 100 \times 0.5+P \times 0.5
$$

Where, both the weight of material output performance and that of people satisfaction performance are 0.5 .

The transformation performance of resource-based cities can be categorized as "Excellent", "Good", "Ordinary" and "Bad", which respectively corresponds with the value of measured satisfaction degree 4.1-5.0, 3.1-4.0, 2.1-3.0 and 0-2.0. The result shows that overall performance of transformation of resource-based Huangshi City is 4.13, which lands in the category of "Excellent".

\section{Conclusions and responsive measures}

\section{Conclusions}

1)The research findings show that Huangshi City has made remarkable achievements in its transformation as a resource-based city. The overall performance is measured as 4.13, which lands in the "Excellent" category, just as expected. Where, in terms of material output performance, the rate of improvement of the economic development indicator achieved the largest value among all the indicators, suggesting that Huangshi City has made remarkable achievement in economic development. However, the indicator of environmental improvement lags behind both the economic development indicator and the livelihood improvement indicator. Therefore, the coordination among economic development, livelihood improvement and environmental improvement still needs to be further strengthened by all the relevant governmental departments with overall coordinated arrangements. As for the three categories of level-one indicators, some of their subordinate indicators still need to be improved. Specifically speaking, the following indicators need to be dealt with decisive measures and increased input of manpower, material resources and policy support so as to safeguard the sound, coordinated and sustainable development of livelihood, environment and economy. in terms of environmental improvement, the ratio of number of days with excellent air quality; in terms of livelihood improvement, the coverage of minimum subsistence guarantee of urban residents; and in terms of economic development, the ratio of R\&D investment to GDP. While, in terms of people satisfaction performance, the satisfaction degree of resident livelihood indicator achieved the largest value among all the indicators, which is generally in consistency with the sound economic condition of the city. However, the satisfaction degree of public service has the least value, which is the very result of incomplete social security system and great employment pressure, two chronic difficulties facing all the resource-based cities.

2)In conclusion, Huangshi City has set up a sound strategy, made a well-conceived deployment of works and executed a thorough implementation of planned measures in its transformation as a resource-based city. However, it still has some indicators that are weak and need to be greatly improved. Therefore, for the future transformation of resource-based cities, governmental departments should make institutional arrangements as per the ordering of value of all the performance indicators, and the performance indicators with low value should be given special 
priority.

\section{responsive measures}

1)In terms of environmental improvement, we should strengthen monitoring and treatment of major pollution sources, strictly screen new projects for environmental assessment, and strengthen environmental supervision and law enforcement.

2)In terms of livelihood improvement, we should strengthen the construction and informatization of employment and business starting service platforms to better serve the employment and business starting of college graduates, migrant workers who return home and new citizens in urban areas.

3)In terms of economic development, we should strive to expand the number of innovative entities and implement preferential policies for innovative enterprises so as to guide all kinds of scientific resources to enterprises, thus increasing the total R \& D input of the society at large.

4)In terms of people satisfaction, we should build a dense livelihood protection network, gradually reduce the gap in basic public services between urban and rural areas, increase the social protection from government and improve the productivity of the agricultural industry so as to enhance the livelihood satisfaction of urban and rural residents and promote the transformation and upgrading of the cities at large.

\section{Acknowledgements}

This work was financially supported by the Hubei Soft Science Research Foundation (2015BDF066,2016ADC080), Young Talent Foundation of Hubei Provincial Department of Education (Q20151512), Science Research Foundation of Wuhan Institute of Technology (K201539) and Foundation of Research Center for Coordinated Development between Enterprises and Environment (G201406).

\section{References}

[1] TANGUAY G A, RAJAONSON J, LEFEBVRE J F, et al. Measuring the sustainability of cities: an analysis of the use of local indicators[J]. Ecological Indicators, 2009, 10(2):407-418.

[2] TURLOUGH F GUERIN. A Survey of sustainable development initiatives in the australian mining and minerals industry[J].Minerals and Energy-Raw Materials Report, 2006, 20(3):11-44.

[3] HILSON G M. Environmental management and sustainable development[J].Journal of Cleaner Production,2006,14(3-4) : 225-226.

[4] SHANG Yun-zhong,WANG Hua-qing. A case study on shanxi province: evaluation of transformation efficiency in resources-based cities[J].Resources \& Industries. 2012,01:12-17.

[5] ZHU Mingfeng, HONG Tianqiu, YE Qiang. Sustainable development indicator forecasting for resource-based cities based on neural network[J]. Journal of University of Science and Technology of China, 2005,35(3):423-428.

[6] WANG Liukai, SHAO Xianwu, HE Yerong, ZHU Yangbao. The study on evaluation factors about transformation of resource-dependent cities based on SEM model[J]. Journal of Huainan Normal University, 2015,17(6):32-35.

[7] CHE Xiaocui, ZHANG Pingyu. Achievement evaluation of economic transformation of resource-based cities based on mutil-quantitative methods[J]. Journal of Industrial Technological Economics, 2011,(2):129-136. 